\title{
Pulmonary atresia, "intact ventricular septum", and aortopulmonary collateral arteries
}

\author{
Reinout J Mildner, Laszlo Kiraly, Narayanswami Sreeram
}

\begin{abstract}
In muscular pulmonary atresia, major aortopulmonary collateral arteries are characteristic of pulmonary atresia with ventricular septal defect and are rarely seen in pulmonary atresia with intact ventricular septum. Two unusual cases of muscular pulmonary atresia are reported, one with an intact septum and one with a perimembranous ventricular septal defect, closed in utero by aneurysmal tricuspid tissue. In both cases the pulmonary blood supply came entirely from aortopulmonary collaterals. In case 1 a collateral artery connected the left subclavian artery and hypoplastic pulmonary arteries, and several aortopulmonary collaterals arose from the descending aorta, without overlap between these two circulations. In case 2 the pulmonary trunk and arterial duct were absent and the pulmonary blood supply came entirely from collateral arteries. The right ventricle was of normal size and tripartite with a closed perimembranous ventricular septal defect, discovered only at postmortem examination. These observations suggest right ventricular outflow tract obstruction early in fetal development, with involution of the pulmonary trunk and sixth arch derivatives, and persistence of primitive aortopulmonary connections. The morphology in case 1 is at odds with the theoretical division of pulmonary atresia with intact septum and pulmonary atresia with ventricular septal defect into two separate pathological entities that occur at different stages in fetal development.
\end{abstract}

(Heart 1997;77:173-175)

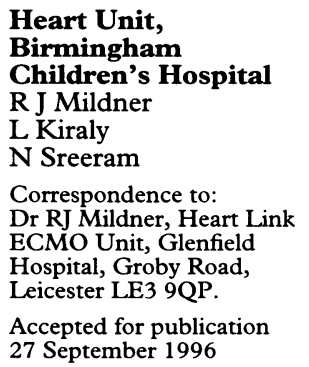

Keywords: pulmonary atresia; intact septum; collateral arteries

Pulmonary atresia with intact ventricular septum (PA/IVS) is a rare cardiac malformation. ${ }^{1}$ The blood supply to the lungs in this disorder is usually dependent on the arterial duct. ${ }^{1}$ In contrast to pulmonary atresia with ventricular septal defect (PA/VSD) major aortopulmonary collateral arteries (MAPCAs) are rarely pre- sent in PA/IVS. We describe two unusual cases: one of PA/IVS and the other of PA/VSD in which the pulmonary blood supply came predominantly from MAPCAs.

\section{Case 1}

A nine month old boy presented with cyanosis from the age of one week. Until assessment, he had lived in India. At physical examination a grade II/VI systolic murmur was heard at the left sternal edge. The diagnosis of PA/IVS was made by echocardiography. However, the communication between the systemic and pulmonary circulations was not identified. Cardiac catheterisation confirmed PA with IVS and suprasystemic right ventricular pressure. The tricuspid valve was hypoplastic (8 $\mathrm{mm}$ diameter) with a small right ventricle, consisting of an inlet portion only. A diminutive pulmonary trunk was present and a right ventriculo-coronary artery connection was demonstrated. An aortopulmonary collateral artery arose from the left subclavian artery to supply confluent but hypoplastic pulmonary arteries. Four further MAPCAs arose from the descending aorta, supplying both lungs (fig $1 \mathrm{~A}-\mathrm{C})$. The aortic arch was right sided and the atrial appendages were juxtaposed. By means of bilateral thoracotomies and a median sternotomy, the right and left sided collaterals were anastomosed to the respective pulmonary arteries, and a $5 \mathrm{~mm}$ central aortopulmonary shunt was created. One hour after surgery he developed evidence of myocardial ischaemia and systemic acidosis. Despite appropriate resuscitation, he died. Postmortem examination confirmed the anatomical diagnosis of muscular pulmonary atresia with intact ventricular septum.

\section{Case 2}

A one day old term, female neonate presented with cyanosis. A continuous murmur, grade II/VI, was heard at the left sternal edge. On echocardiography, the right ventricle was well formed (tricuspid valve $11 \mathrm{~mm}$ diameter), but the pulmonary trunk or arteries could not be identified and the ventricular septum appeared intact. An arterial duct was not seen. At emergency cardiac catheterisation the provisional diagnosis of PA/IVS was made. The tricuspid 
Figure 1 (A) Left subclavian arteriogram (patient 1) showing collateral artery supplying confluent but hypoplastic native pulmonary arteries. LP, left pulmonary artery; $R P A$, right pulmonary artery. (B) Descending aortogram (patient 1) showing collateral arteries (CO) supplying both lungs. (C) Composite diagram showing the parenchymal distribution of native pulmonary arteries and collateral arteries in patient 1. Aorta (unshaded), pulmonary trunk and arteries (light shade); collateral arteries (dark shade). Desc Ao, descending aorta; $M P A$, main pulmonary artery.
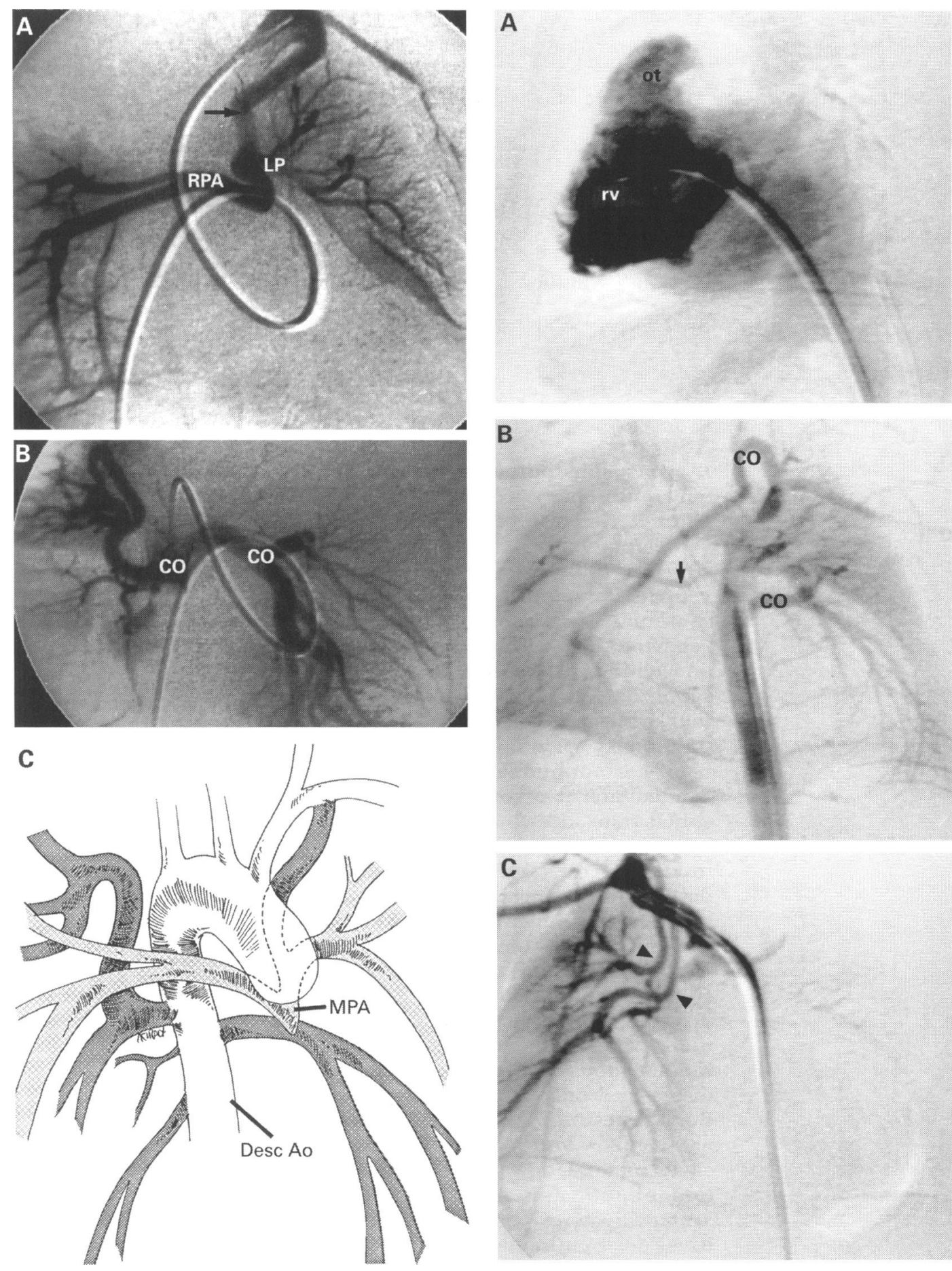

valve was only mildly regurgitant; the right ventricle was well developed and tripartite (fig 2A) with suprasystemic right ventricular pressure. The pulmonary trunk was absent, the native pulmonary arteries were severely hypoplastic but confluent (fig 2B), and the arterial duct was absent. Several collateral arteries from the brachiocephalic trunk supplied the upper part of the right lung (fig 2B). The lower lobe of the right lung was supplied from a collateral artery arising from the left subclavian artery. Further direct MAPCAs arose from the descending aorta supplying the left lung (fig 2B). Coronary artery distribution was normal. In view of the severe hypoplasia of the native pulmonary arteries and the small size of individual collateral arteries, it was decided not to undertake surgical palliation.
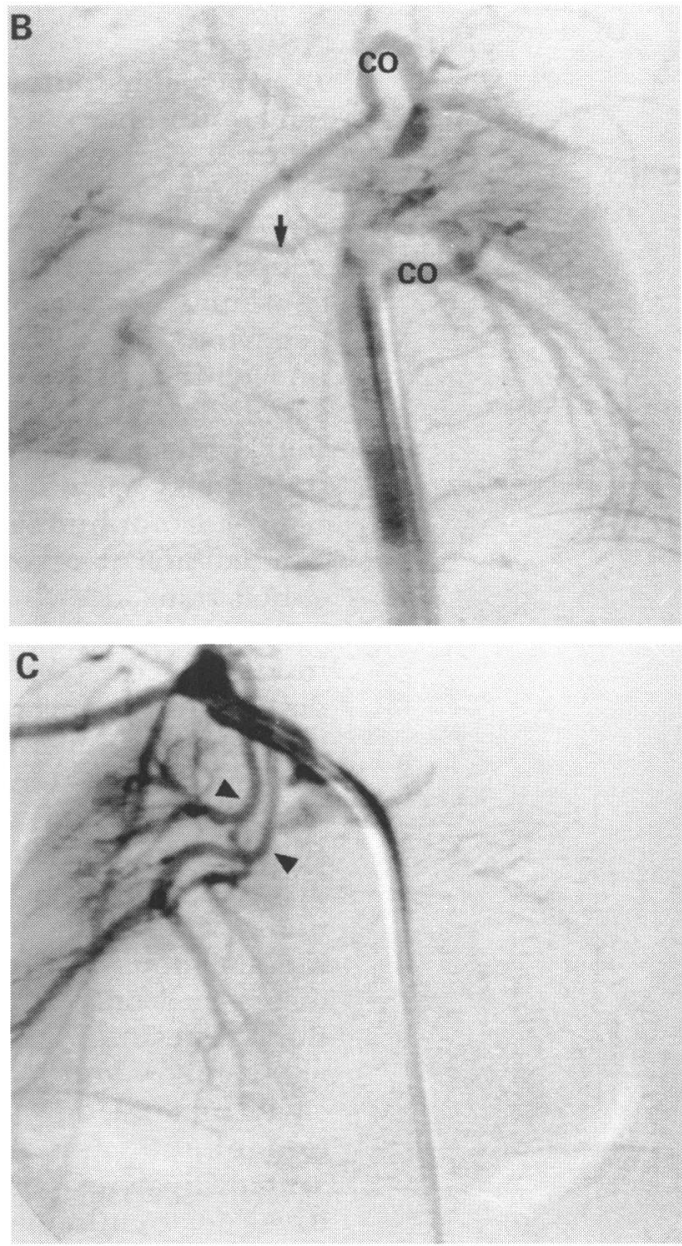

Figure 2 (A) Right ventriculogram (patient 2) showing tripartite right ventricle ( $r v$ ) with mild tricuspid

regurgitation. ot, outflow tract. (B) Aortogram in patient, showing collateral arteries (CO) supplying left lung and lower lobe of right lung. Arrow indicates confluent, hypoplastic pulmonary arteries. (C) Brachiocephalic arteriogram in patient 2 , showing two collateral arteries (arrows) supplying the upper segments of the right lung.

The patient died suddenly at home aged one month. At postmortem examination muscular pulmonary atresia was confirmed, with absent pulmonary trunk. However, there was a small perimembranous VSD which was closed by tricuspid valve tissue and had not been noted on angiography. This led to the revised diagnosis of PA/VSD. 


\section{Discussion}

Pulmonary blood supply via MAPCAs is commonly associated with PA/VSD and occasionally with tetralogy of Fallot. Only very rarely has it been described in other types of pulmonary atresia, including PA/IVS. ${ }^{1}$

De Ruiter et $a l^{2}$ suggested that in early embryonic development transient systemic to pulmonary collateral arteries may arise during two extended periods. During maldevelopment of the pulmonary outflow tract these transient connections may persist as systemicpulmonary collateral arteries. The timing and the extent of pulmonary outflow tract maldevelopment may determine the origin and distribution of the collateral arteries. In addition these workers postulate that when pulmonary obstruction occurs at a much later stage of fetal development or postnatally, bronchial arteries may develop into systemic-pulmonary collateral arteries. Thiene $e t a l^{3}$ found that in PA/VSD, early right ventricular outflow tract obstruction leads to involution of the pulmonary trunk and sixth aortic arch derivatives with persistence of primitive aortopulmonary connections.

Our two cases show several features usually associated with PA/VSD. Both patients had muscular pulmonary atresia with an absent or diminutive pulmonary trunk that is more commonly seen in PA/VSD. ${ }^{4}$ In addition a normal arterial duct was absent in both, with the pulmonary blood supply coming entirely from MAPCAs. In case 1 the anatomical findings can be explained if it is assumed that a VSD which was present during early fetal life had subsequently closed. But without evidence for such a feature, the combination of PA/IVS with pulmonary blood supply by MAPCAs is at odds with the division of pulmonary atresia with intact septum and pulmonary atresia with
VSD into two separate pathological entities. ${ }^{5}$ In case 2 the presence of a VSD closed by aneurysmal tricuspid tissue explains the finding of a well developed, tripartite right ventricle. This observation is supported by Orie et $a l^{6}$ who found that most isolated VSDs diagnosed antenatally close in utero. Therefore in cases of apparent PA/IVS with MAPCAs the possibility of in utero closure of a VSD should be considered.

Kutsche and Van Mierop and Santos et al ${ }^{57}$ reported that in PA/IVS the arterial duct is usually well developed and has a specific morphology suggesting anterograde flow until late fetal gestation. Case 1 was unusual in this respect because there was no characteristic arterial duct. However, we have no data on the histology of the collateral arteries arising from the subclavian arteries in either case to establish whether these are in fact true collaterals or arterial ducts.

1 Freedom RM, Burrows PE, Smallhorn JF. Pulmonary atresia and intact ventricular septum. In: Freedom RM, Benson LN, Smallhorn JF, eds. Neonatal heart disease. Benson LN, Smallhorn JF, eds. Neonatal

2 De Ruiter MC, Gittenberger-de Groot AC, Poelmann RE, De Ruiter MC, Gittenberger-de Groot AC, Poelmann RE,
Van Ieperen L, Mentink MMT. Development of the pharyngeal arch system related to the pulmonary and bronchial vessels in the avian embryo. With a concept on systemic-pulmonary collateral artery formation. Circulation 1994;87:1306-19.

3 Thiene G, Frescura C, Bortolotti U, Del Maschio A, Valente $M$. The systemic pulmonary circulation in pulmonary atresia with ventricular septal defect: concept of fourth and sixth aortic arches. Am Heart $\mathcal{F} 1981 ; 101$ : 339-44.

4 Elzenga NJ, Gittenberger-de Groot AC. The ductus arteriosus and stenosis of the pulmonary arteries in pulmonary atresia. Int $f$ Cardiol 1986;11:195-208.

5 Kutsche LM, Van Mierop LHS. Pulmonary atresia with and without ventricular septal defect: a different etiology and pathogenesis for the atresia in the 2 types? $A m$ Cardiol 1983;51:932-5.

6 Orie J, Flotta D, Sherman FS. To be or not to be a VSD. Am f Cardiol 1994;74:1284-5.

7 Santos MP, Moll JN, Drumond C, Arujo WB, Romao N, Reis NB. Development of the ductus arteriosus in right ventricular outflow tract obstruction. Circulation 1980;62: 818-22. 